Paranoiac-Critical Method. His central idea was that paranoia is essentially a mental mechanism which can be used by the artist in a controlled way to create artistic images. Dali used the term 'paranoia' in the broad sense of a 'delusion of interpretation'. The notion of the 'persecution complex' was not essential to the definition. For him the paranoid state could be induced voluntarily and involved the subject perceiving images, ideas or events as being causally connected or as being all related to one central idea. These perceptions are internally coherent for the subject of the delusion but without meaning for an observer. Paranoiac-critical activity is a 'spontaneous method of irrational knowledge based upon the interpretive-critical association of delusional phenomena' ${ }^{8}$ This 'irrational knowledge' could be made 'concrete' by its incorporation in paintings. Examples of this include the many images with a double configuration which are found in Dali's paintings.

These are only some of the themes common to surrealism and psychoanalysis and psychiatry. Freud was by far the greatest intellectual influence on the surrealist movement. Developments in psychoanalytic technique were mirrored by developments in surrealist artistic techniques. In their productions one can often determine the influence of analytic and psychiatric concepts and classifications.
While surrealism has never had a great influence on the art and literature of these islands, it would be difficult to quantify its impact on continental and American culture. Psychoanalysis has played an increasingly important role in French psychiatry since 1968. A reexamination of surrealist concepts has also occurred. It would be a pity if psychiatrists in the UK failed to appreciate the wealth of 'psychological' interest offered by surrealist art to the interested observer.

\section{REFERENCE}

'LA ReVolution SurRÉliste (1984) An historical vignette: surrealism and anti-psychiatry. Bulletin of the Royal College of Psychiatrists, 8, 175.

${ }^{2}$ BALAKIAN, A. (1972) Surrealism: The Road to the Absolute. Page 131. London: Unwin Books.

${ }^{3}$ Breton, A. (1969) Manifestoes of Surrealism. Page 14. University of Michigan Press.

4_ (1969) Manifestoes of Surrealism. Page 160. University of Michigan Press.

AdDes D. (1982) Dali. Page 121. London: Thames \& Hudson.

${ }^{\circ}$ Breton, A. (1978) What is Surrealism? Page 49. London: Pluto Press.

'ADEs, D. (1982) Dali. Page 82. London: Thames \& Hudson.

- (1982) Dali. Page 126. London: Thames \& Hudson.

\title{
The Premenstrual Syndrome
}

\author{
Gwyneth A. SAmpson, Middlewood Hospital, Sheffield
}

A conference on the premenstrual syndrome (PMS) was held in September 1984 in Philadelphia, USA, jointly supported by the Ethics and Values in Science and Technology (EVIST) Section of the National Science Foundation, and by a grant from the National Endowment for the Humanities (USA). Twenty-two international research workers in the area of PMS met for a week's closed workshop discussing the scientific, legal and ethical issues raised by the present attention being given to the syndrome. PMS is now popular in the USA, with many private clinics and programmes being developed. Ten of the twenty-two invited participants were from medicine (six of these were psychiatrists, two general practitioners and four gynaecologists); the remainder were sociologists, anthropologists, lawyers, physiologists, philosophers, behavioural geneticists, psychologists, social workers, criminologists and bioethicistsan indicator of the diversity of the effects of and research into premenstrual syndrome. There were three UK participants - a general practitioner, a gynaecologist, and a psychiatrist. As the UK psychiatrist I felt that two in particular of the concensus opinions reached by such a diverse group would be of interest to readers of the Bulletin.
The subgroup from those workers involved with Social Science Research and Public Policy reported:

The menstrual cycle is a normal event in the lives of most women of reproductive age. It does not disrupt their behaviour and does not disrupt their lives. A small percentage of women experience recurring menstrual-related problems, and some of these women find that those problems seriously affect their lives. Despite cases claiming an association between violent acts and the menstrual cycle, there are no studies confirming this. It is a violation of the dignity of all women to perpetuate stereotypes about the effect of menstruation on behaviour.

Regardless of aetiology, the experience of cyclic symptoms is an important and sometimes a serious one. We need to recognise women's experiences of these recurring symptoms and not dismiss them as figments of their imagination. At the same time, we need to distinguish these problems from other problems that have been mistakenly called PMS.

Unfortunately, the nature of the phenomenon called PMS remains ill-defined. Various aspects of the menstrual cycle need further interdisciplinary investigation. Psychological research shows clearly that using self-reports results in biases of various sorts. Social and cultural factors influence women's reported experiences and memories of them. This conclusion is shown by the research indicating that women's reported experiences vary as a 
function of culture, religion, social class, socialisation at menarche, and current life events.

Because the aetiology of this phenomenon is unknown, all treatments are experimental. No treatment is risk free. We urge caution and consideration of risks and benefits on the part of both health care professionals and people seeking treatment. We are concerned about the commercialisation of treatments based upon limited and inconclusive evidence.

To correct the widespread misunderstanding of menstrual cyclerelated experience, education should be a top priority. We recommend creative education programmes directed toward the general public, including both children and adults. In addition, special attention should be paid to the training and continuing education of members of the biomedical community. Because of their perspective, women's groups should be centrally involved in these programmes.

\section{The Biomedical Subgroup reported:}

Many women report a variety of physical and behavioural changes that occur in temporal relation to the menses, but there is, at present, no universally accepted definition of PMS. Although a number of symptoms such as irritability, abdominal bloating, mood lability, breast tenderness, hostility, and tension are frequently reported in relation to the premenstruum, diagnosis of PMS cannot be made solely on the basis of symptomatology. Most experts agree that a precise definition of PMS must incorporate information about severity and timing of symptoms. The importance of obtaining this information prospectively cannot be overemphasised. Prospective records have, in fact, revealed the existence of a group of women in whom symptoms cyclically reappear in the premenstrual phase of the cycle, with relief following the onset of menstruation. At times, premenstrual symptoms are sufficiently distressing to result in reports of significant impairment of function. However, it is not yet known if PMS is an extreme form of formal premenstrual symptoms, or if it is a distinctive clinical entity.

The current uncertainty arises in part from the differences that can be expected when separate disciplines examine a subject and from the inherent difficulty in comparing work that uses widely disparate constructs. It is even questionable whether various investi- gators have been examining the same basic phenomenon. Much of the confusion may result from the tendency to generalise findings from restricted samples of women.

Many modalities have been reported to be effective in the treatment of PMS. However, evidence at present does not support any currently aetiological model, nor does it support the therapeutic superiority of any prescribed treatment. The cornerstone of the treatment of PMS at present should be careful, comprehensive, longitudinal evaluation with attention paid to the severity and pattern of appearance of symptoms, as well as to medical, social, and psychological factors that may affect symptom appearance. Treatment may include such modalities as social support, education, counselling, life-style changes, and pharmacotherapy tailored to a patient's individual needs.

Much of the present difficulty in assessing treatments results from methodology that omits necessary control and placebo conditions, as well as the failure to select patients/subjects on the basis of multiple prospective ratings throughout the menstrual cycle and over the course of several cycles. Further conclusions concerning the efficacy of treatment modalities must await the results of rigorously designed studies that include the following: utilisation of clear and consistent definitions of PMS; prospective diagnostic confirmation; double-blind placebo control trials; utilisation of treatment cells that are long enough to avoid the obfuscating effects of intercycle symptomatic variation; and consideration of socio-cultural variables. In addition, collateral objective assessment of symptoms is recommended to evaluate the reliability of subjective ratings.

It appears that the least ambiguous results will be obtained by studies of women who are severely affected premenstrually yet have no clinical evidence of emotional or behavioural disturbance at other phases of their menstrual cycle. If PMS could be defined in these women and the data used to construct operationally-defined criteria and specific symptom-rating tools, it should be possible to compare more reliably future studies of the aetiology and treatment of this disabling condition.

Currently available data do not permit reliable estimates of the prevalence and impact of this disorder, highlighting the importance of the need for well-designed, prospective, epidemiological studies.

\section{Correspondence}

\section{The adolescent services}

DeAr SiRs

Dr Perinpanayagam's letter (Bulletin, November 1985, 9, 226-227) makes reference to those of us working in Child Guidance settings as not having opportunities to develop expertise in working with adolescents. May I assure him that this is not the case in all Child Guidance Clinics. Here in Macclesfield, although the title has been retained for historical reasons, over a third of our Child Guidance referrals are aged 12 and over and include a number of youngsters deemed 'unsuitable' for treatment by adolescent in-patient services.

\section{Child Guidance Clinic \\ Wilmslow Road, \\ Handforth, Cheshire}

DiANNe M. BURTon 